\title{
Correction to: NIX protein enhances antioxidant capacity of and reduces the apoptosis induced by HSP90 inhibitor luminespib/NVP-AUY922 in PC12 cells
}

\author{
Hong Zhang ${ }^{1} \cdot$ Fanghui $\mathrm{Ge}^{1} \cdot$ Xindong Shui ${ }^{2,3} \cdot$ Yuling Xiang $^{2,4} \cdot$ Xinxin Wang ${ }^{1} \cdot$ Chang Liao $^{1} \cdot$ Jiandong Wang ${ }^{2,5}$
}

Accepted: 8 June 2021 / Published online: 1 July 2021

(C) Cell Stress Society International 2021

\section{Correction to: Cell Stress and Chaperones (2021) 26:495-504 https://doi.org/10.1007/s12192-021-01193-6}

In the above mentioned publication, the online supplementary information (ESM 1) contained an error in supplementary Fig. 4. The original article has been corrected.

\section{Supplementary Information The online version contains supplementary material available at https://doi.org/10.1007/s12192-021-01218-0.}

Publisher's note Springer Nature remains neutral with regard to jurisdictional claims in published maps and institutional affiliations.

The online version of the original article can be found at https://doi.org/ 10.1007/s12192-021-01193-6

Jiandong Wang

wangjiandong79@cmc.edu.cn

1 School of Laboratory Medicine/Sichuan Provincial Engineering Laboratory for Prevention and Control Technology of Veterinary Drug Residue in Animal-origin Food, Chengdu Medical College, Chengdu 610500, Sichuan, China

2 School of Bioscience and Technology, Chengdu Medical College, Chengdu 610500, Sichuan, China

3 School of Basic Medical Sciences, Fujian Medical University, Fuzhou 350122, Fujian, China

4 College of Life Science and Technology, Guangxi University, Nanning 530004, Guangxi, China

5 Collaborative Innovation Center of Sichuan for Elderly Care and Health, Chengdu Medical College, Chengdu 610500, Sichuan, China 